\title{
A Case Report: Convulsion and Reduced Level of Consciousness in Two Children Following Liquefied Petroleum Gas Inhalation
}

Nasiri Jafar ${ }^{2}$, Hashemi Simin² and Sadinejad Mortaza ${ }^{1 *}$

${ }^{1}$ Department of Pediatric Neurology, Faculty of Medicine, Isfahan University of Medical Sciences, Isfahan, Iran

${ }^{2}$ Child Growth and Development Research Center, Isfahan University of Medical Sciences, Isfahan, Iran

\begin{abstract}
Liquefied Petroleum Gas (LPG) is widely used as fuel at homes and in vehicles in the compressed gas cylinders. Previously, some reports of toxicity with gas inhalation causing different symptoms from a simple headache and dizziness to coma and death had been published. In this report, the states of 2 children, a 5-year-old girl and her 2-yearold brother, who developed convulsion and reduced level of consciousness following playing with and opening the gas valve of a portable cooking gas stove at the kitchen, are explained.
\end{abstract}

Keywords: Liquefied petroleum gas; Hydrocarbons; Parkinsonism

\section{Introduction}

LPG is a mixture of aliphatic hydrocarbons widely used as fuel in the compressed gas cylinders at home and in vehicles. It is a mixture of combustible gases including $20 \%$ propane and $80 \%$ butane. Numerous cases of poisoning with this gas have been published [1].

There are several cases of sudden death after inhaling LPG mainly caused by its incomplete combustion. A few cases of accidental or intentional poisoning with LPG before combustion have been reported to lead to various complications from simple mucosal irritation to Parkinsonism and death $[2,3]$.

\section{Case Report}

In this report, two children (a 5-year-old girl and her 2-year-old brother) are described who have been poisoned due to inhalation of LPG gas and subsequently developed convulsion and reduced level of consciousness while playing with and opening the gas valve of a portable cooking gas stove at the closed space of the kitchen for nearly 1 hour.

The baby boy had reduced level of consciousness and several episodes of generalized tonic clonic seizure before being referred to the healthcare center. The baby was not conscious on arrival and rather in an agitated confusional state. The patient had periorbital edema and mildly increased bilateral DTR. No skin lesion was observed.

He was referred to our medical center after receiving phenytoin at the previous healthcare center. On arrival, naloxone was administered for the patient, but no dramatic response was observed. In his blood test, no special finding was observed except for a slight increase in AST. ECG was normal. vital signs were normal. In the urine toxicology, no evidence of poisoning with opioids or any other drugs was found. Chest X ray was normal. His brain CT scan and MRI were also normal. Supportive care and phenytoin were continued. During the next 12 hours, state of consciousness gradually increased. The next day, the patient was alert and calm, while his vital signs were stable.

Similarly, the baby girl had reduced level of consciousness and one episode of seizure as GTC before being admitted to the emergency department. The patient was unconscious on admission to our center. Naloxone was administered on his arrival but no dramatic response was observed. Moreover, no findings were discovered in ECG and CXR and his brain MRI and CT scan were normal as well. The clinical examination demonstrated a reduced level of consciousness and the rest of physical examinations were normal. Phenytoin was started and continued for the patient. The next day, the patient was alert and calm and his vital signs were stable.

\section{Discussion and Conclusion}

Intentional and accidental inhalations of LPG with the main gas contents of propane and ISO butane have increased recently. These gases are generally considered to be safe. In a study on LPG, no toxic effects of the gas were observed within the concentrations of 9000-16000 PPM [1]. However, a number of reports have been published on the LPG toxicity. Probably, inhalation of toxic gases such as carbon monoxide due to incomplete combustion causes some cases of poisoning when using this gas [2].

Yet, there are rare reports that the gas has led to poisoning and various complications in its pure form prior to combustion. In an autopsy report of two workers involved in hypoxia and death following inhalation of LPG when working with gas pipes the liquid amounts of propane gas (LPG main component) were found to be 0.12 and 3.40 $\mathrm{mg} / 100 \mathrm{~g}$ [3].

Furthermore, there are some reports of poisoning with LPG leading severe ataxia, bradykinesia, dystonia, dysarthria, and arrhythmias [3] In the previous reports, massive rhabdomyolysis has been followed by LPG inhalation [4]. As for the 2 patients described in this report, they had been poisoned after inhaling the gas due to opening the gas cylinder valve. These hydrocarbons are lipophilic and it seems that after inhalation into the lungs and being absorbed into the blood, they enter fat-rich tissues (especially brain and fat tissues) and cause neurological symptoms including reduced level of consciousness, convulsion, ataxia, etc. [5].

It seems despite having low toxicity, such gases may cause a direct

*Corresponding author: Sadinejad Mortaza, Department of Pediatric Neurology, Faculty of Medicine, Isfahan University of Medical Sciences, Isfahan, Iran, Tel: +983137923071; E-mail: sadinejad@med.mui.ac.ir

Received February 12, 2017; Accepted March 06, 2017; Published March 09 2017

Citation: Jafar N, Simin H, Mortaza S (2017) A Case Report: Convulsion and Reduced Level of Consciousness in Two Children Following Liquefied Petroleum Gas Inhalation. J Environ Anal Toxicol 7: 444. doi: 10.4172/2161-0525.1000444

Copyright: @ 2017 Jafar N, et al. This is an open-access article distributed under the terms of the Creative Commons Attribution License, which permits unrestricted use, distribution, and reproduction in any medium, provided the original author and source are credited. 
Citation: Jafar N, Simin H, Mortaza S (2017) A Case Report: Convulsion and Reduced Level of Consciousness in Two Children Following Liquefied Petroleum Gas Inhalation. J Environ Anal Toxicol 7: 444. doi: 10.4172/2161-0525.1000444

Page 2 of 2

damage to the brain through hypoxic mechanism. In the case of our patients, the gas cylinders containing LPG had been off and the children had been poisoned when playing with them due to opening their valves and direct inhalation of LPG [6]. This study is important in this respect that clearly shows that poisoning by pure LPG inhalation can cause neurological damage manifesting as reduced level of consciousness and convulsion. At present, it is not clear whether the symptoms of poisoning with such gases are related to their direct effects on the brain or the brain hypoxia can lead to the symptoms of toxicity.

\section{References}

1. McKee RH, Herron D, Saperstein M, Podhasky P, Hoffman GM, et al. (2014) The toxicological properties of petroleum gases. International journal of toxicology 33: $28 \mathrm{~S}-51 \mathrm{~S}$
2. From the Centers for Disease Control and Prevention (2000) Carbon monoxide poisoning associated with use of LPG-powered (propane) forklifts in industrial settings--lowa, 1998. JAMA 283: 331-332.

3. Fukunaga T, Yamamoto H, Tanegashima A, Yamamoto Y, Nishi K (1996) Liquefied petroleum gas (LPG) poisoning: report of two cases and review of the literature. Forensic Science International 82: 193-200.

4. Prasad S, Singh R, Manocha R, Narang M, Sharma BD, et al. (2009) Acute massive rhabdomyolysis due to inhalation of LPG. JAPI 57: 472-473.

5. Godani M, Canavese F, Migliorini S, Del Sette M (2015) Ataxia with Parkinsonism and dystonia after intentional inhalation of Liquefied Petroleum Gas. Neuropsychiatric Disease and Treatment 11: 1157.

6. Pfeiffer H, Al Khaddam M, Brinkmann B, Köhler H, Beike J (2006) Sudden death after isobutane sniffing: a report of two forensic cases. Int J Legal Med 120: 168-173. 